\title{
Multisystem Langerhans Cell Histiocytosis With Hypothyroidism and Sclerosing Cholangitis: a Case Report and Literature Review
}

\section{Xiaoyan Liu}

shandong public health clinical center

Shuangshuang Xie

shandong public health clinical center

Wenjun Du ( $\nabla$ crbdwj@126.com )

shandong pubilc health clinical center

\section{Case report}

Keywords: Langerhans cell histiocytosis, Sclerosing cholangitis, Skin, Thyroid, Nails, Erythrocyte sedimentation rate

Posted Date: May 4th, 2021

DOI: https://doi.org/10.21203/rs.3.rs-439626/v1

License: (c) (i) This work is licensed under a Creative Commons Attribution 4.0 International License. Read Full License 


\section{Abstract}

Background: Langerhans cell histiocytosis (LCH) is a rare disease caused by a disorder of dendritic cells. $\mathrm{LCH}$ mainly occurs in children and the tissue damage is caused by proliferative dendritic cells. In most LCH patients, only a single organ is affected, whereas some cases may involve multiple organs, such as bone marrow, liver, lungs, and brain.

Case presentation: Herein we present an LCH case, that involved a multisystem: the skin, lungs, thyroid, liver, nails, and spleen. Low appetite, fatigue, and jaundice were the main symptoms. The patient had extensive skin lesions, nail lesions, macular sclera, and bilateral goiter. The erythrocyte sedimentation rate (ESR) was higher than normal. Lymphocytic thyroiditis was diagnosed by fine needle biopsy and skin rash. A liver biopsy revealed sclerosing cholangitis (SC). Response to treatment with Etoposide®VP-16), Prednisone acetate tablets, cyclophosphamide, and vinblastine was excellent. With disease remission, the corresponding ESR levels decreased.

Conclusions: LCH involved not only the skin and thyroid, but also liver, lungs, nails and possibly the spleen in this case. Currently there are no standard treatment options and the treatment needs to be individualized. It is rare that $\mathrm{LCH}$ with sclerosing cholangitis is the first presentation, and we should be vigilant to whether other organs are involved. An elevated ESR may be a valuable indicator of disease activity in patients with Langerhans cell histiocytosis.

\section{Background}

Langerhans cell histiocytosis (LCH) is a clonal proliferative disorder of the dendritic ( Langerhans) cells (1). The clinical manifestations depend on the involved sites and organs, such as lymph nodes, hypothalamus, pituitary gland, lungs, bones, spleen, liver, and thyroid. As a result, its clinical symptoms may vary and depend on the involved organs or lesion size. More than $60 \%$ of LCH patients have singlesystem disease, but in patients with multisystem disease, the incidence of skin and lung involvement was reported as $50 \%$ and $20 \%-50 \%$, respectively (2). LCH is a disease with obvious heterogeneity, which can manifest as an isolated lesion of a single organ system or a highly disseminated malignant process, and the prognosis can be natural remission or deterioration.

Cytopenia is observed more frequently in patients with multiple site LCH than those with single system $\mathrm{LCH}$, and histiocytosis and an occasional megakaryocyte decrease in the bone marrow occurs in $10-15 \%$ of patients (3). Ultrasound examination can find liver, spleen, thyroid, thymus, and other swellings. Large histiocytes with abundant cytoplasm interspersed in a background of lymphocytes and eosinophils are the typical and the first presentation of LCH by light microscopy (2). The Histocyte Society recommended that a presumptive diagnosis of LCH should be made based upon light microscopic findings and a compatible clinical picture, but a definitive diagnosis requires that lesion cells exhibit positive staining with S-100 and CD1a. Although the "gold standard" for identification of Langerhans cells has been the 
detection of Birbeck granules by transmission electron microscopy, this technique is rarely used today with limited numbers seen in involved organs (1).

Presentation of the liver involving Langerhans cell histiocytosis can be variable. Infiltration of the intraand extra-hepatic biliary tree, sclerosing cholangitis, hepatomegaly, hepatic heterogenous node, and hepatic fibrosis were reported (4-6). Among the liver involvement, the incidence of sclerosing cholangitis in $\mathrm{LCH}$ ranges from 10 to $18 \%$ (7). When $\mathrm{LCH}$ presents with SC, $\mathrm{LCH}$ generally involves other systems, which means that SC may be a complication of disseminated LCH. In this case, the patient's liver biopsy implied SC. After confirmation by skin and thyroid biopsies, we diagnosed LCH associated with secondary sclerosing cholangitis.

\section{Case Presentation}

A 20-year-old male patient was admitted into the liver disease department with complaints of low appetite, fatigue, and jaundice for more than one year. Physical examination found extensive coalescing, scaling, or crusted papules on his skin (Fig. 1A口B). Onycholysis, loss of nail plates, and subungual hyperkeratosis were seen in most of his fingernails (Fig. 1CDD). Yellow-stained sclera was also found. The bilateral thyroid was tumescent, while the liver and spleen were not palpable under the cost arch. He had a medical history of 4 years lymphocytic thyroiditis diagnosed by fine needle aspiration biopsy and skin rashes for more than 3 years. A year previously, his nails began to keratinize. He was taking Levothyroxine Sodium (Euthyrox) for his Hashimoto's thyroiditis. A family history was negative for rheumatic or inherited liver diseases.

Laboratory examination results showed that the complete blood cell count was within normal limits, with a high erythrocyte sedimentation rate $(\mathrm{ESR})$ that was $55 \mathrm{~mm} / \mathrm{h}(\mathrm{NV}<15)$, cholestasis-alkaline phosphatase 456U/L $(\mathrm{NV}<126)$ and GGT $189 \mathrm{U} / \mathrm{L}(\mathrm{NV}<85)$, total bilirubin $184.99 \mathrm{umol} / \mathrm{I}(\mathrm{NV}<17.1)$, direct bilirubin150.76 umol/l, aline aminotransferase $18 \mathrm{U} / \mathrm{L}(\mathrm{NV}<40)$, while T3 and T4 were within the normal range, but TSH was $14.88 \mathrm{UIU} / \mathrm{ml}(\mathrm{NV}<6)$. Hepatitis $B$ virus surface antigen as well as hepatitis $\mathrm{C}$ antibodies and human immuno-deficiency virus antibody were negative. Abdominal ultrasonography revealed an enlargement of the liver and spleen, while no ascites were detected. Liver biopsy detected the formation of bile pigment granules, an irregular intrahepatic duct wall, small bile duct proliferation, and periductal fibrosis, which implied the diagnosis of sclerosing cholangitis (SC) (Fig. 2A]B). However, we could not confirm the diagnosis of SC with the results showing the negative autoimmune antibody with the elevated IgG and ESR. After one week, the skin pathology predominantly consisted of macrophages and positivity to CD1a surface antigen and S-100 protein, leading to the diagnosis of LCH (Fig. 2C口D). However, immunohistochemical analysis of liver tissue showed negative staining for CD1a and S100 with no typical portal area in the following slice.

A CT scan of the head, chest, and abdomen were done to detect involvement of other organs. The CT head scan did not reveal any lytic lesions. The CT scan of the lungs showed diffuse small multiple lung cysts. The CT scan of the abdomen confirmed the findings of sonography with hepatomegaly and 
splenomegaly, indicating the involvement of these organs (Fig. 1EロF). A brain MRI did not reveal any acute abnormality. The patient also underwent pulmonary function testing with no obstructive or ventilation abnormalities. A fine needle aspiration biopsy of the thyroid confirmed the diagnosis of $\mathrm{LCH}$ with positive CD1a and S100 immunohistochemical staining(Fig. 2E₫F).

Based on the diagnosis of $\mathrm{LCH}$ involving the skin, thyroid, liver, lungs, and other organs, the patient was treated with Etoposide and prednisolone, ursodeoxycholic acid (UDCA) for his cholestasis, and Euthyrox for hypothyroidism. After 6 months of treatment, he had a worsening of the skin rash and an increase in size of the thyroid with no significant decrease in ESR, so the treatment was changed to a combination of chemotherapy with VP-16, Prednisone acetate tablets, cyclophosphamide, and vinblastine. The patient achieved complete remission after 12 months of chemotherapy with supportive treatment. After 12 months treatment, skin lesions and nails changes cleared-up, the thyroid function was normal, and jaundice was resolved with a decrease in ESR.(Table 1)

For this study we obtained informed written consent from the patient authorizing publication of his clinical case and his photographs. His anonymity has been preserved.

\section{Discussion And Conclusions}

We presented a patient who suffered from multisystem LCH with hypothyroidism and sclerosing cholangitis. He was initially diagnosed and treated for cholestasis but the results of biopsy showed the infiltration of Langerhans cells in the skin and thyroid tissues. The patient had been diagnosed and treated for Hashimoto's thyroiditis for thyroid hypermegaly three years previously. Further examinations found that LCH also involved his lungs, some nails, and spleen.

$\mathrm{LCH}$ can involve any organ system in the body, but the frequency of involvement depends on age(2). Several large retrospective studies consisting of neonates and children under the age of 4 years, showed that $51-71 \%$ of children with LCH present with multisystem disease, but $69-72 \%$ of adults only involve a single organ(3). A recent report from the International Registry of the Histiocyte Society showed that single-system Langerhans cell histiocytosis was found in $31.4 \%$ of patients, $68.6 \%$ had multisystem disease, and $29.6 \%$ had diabetes insipidus $(3-4,8)$. Among the multisystem lesions, the involvement of lungs, bones, and skin are common (9). As a result, when a patient shows a multisystem disease, we should be aware of $\mathrm{LCH}$, and if we cannot diagnose the disease according to one organ lesion, we should seek clues from other organs. In this case, the skin biopsy prompted us to the diagnosis of LCH. As a clue to the disease, the following biopsy of the thyroid also reinforced the diagnosis of LCH. Finally, we concluded that the disease involved not only skin and the thyroid, but also liver, lungs, nails and possibly the spleen. A case involving so many organs is rare, especially interfering with the liver, nails, and thyroid.

Because reports about $\mathrm{LCH}$ are still limited to case reports, the etiology of $\mathrm{LCH}$ must remain speculative. However, it has been suggested that abnormal Langerhans cells elucidate cytokines, such as the plateletderived growth factor, interleukin 1 , and tumor necrosis factor, which may play an important role in the development of hepatic fibrosis (5). This is also presumed to be the mechanism of multisystem clonal 
infiltration. In our case, the ESR was higher than normal, indicating the active role of the immune reaction and activity of the disease. With disease remission, his ESR level decreased. Calming et al reported that ESR estimates $(n=76)$ associated with active disease was $21 \mathrm{~mm} / \mathrm{h}(S D \pm 68.4)(10)$. An elevated ESR may be a clinically valuable indicator of disease activity in patients with $\mathrm{LCH}$, but the role of ESR, nail, and thyroid involvement as poor prognostic signs are unclear.

During the treatment, the patient did not respond well to $\mathrm{Vp}-16$ and prednisolone chemotherapy. During tapering, the rashes and liver function of the patient relapsed. Then the chemotherapy strategy was changed to VP-16, Prednisone acetate tablets, Cyclophosphamide, and vinblastine following the recommendation of a hematologist. Though the patient developed adverse events such as bacterial infection, combination chemotherapy continued with supportive treatment. According to a report, VP-16 treatment is less efficacious to multisystem LCH. From this case, we learned that primarily using a stronger combination chemotherapy is beneficial and the VP-16 combination with Prednisone acetate tablets is not the best choice in such cases.

The disadvantages of this study are lack of checking of the BRAF V600E mutation and that the follow-up time was relatively short. Previous study reported that the BRCA V600E mutation is related to multiple system involvement, high risk, and relapse of $\mathrm{LCH}$. We will collected a large amount of laboratory data to clarify the correlation between BRAF V600E mutation and ESR changes in LCH patients, and the clinical manifestations and disease prognosis of each system.

Reported LCH related SC progresses more rapidly than other forms of sclerosing cholangitis. Most LCHrelated SC patients received liver transplantations at the late stage of fibrosis that is effective (11-13). $\mathrm{LCH}$ reoccurrence after transplantation is relatively low. Involvement of the thyroid and nails in multisystem LCH is a sign of poor prognosis. Based on the poor prognostic features of this case, we decided to treat with more aggressive regimens as we thought that might be an effective option of treatment for multisystem $\mathrm{LCH}$.

\section{Abbreviations}

LCH: Langerhans cell histiocytosis; ESR: Erythrocyte sedimentation rate; SC: Sclerosing cholangitis: UDCA: Ursodeoxycholic acid

\section{Declarations}

\section{Ethics approval and consent to participate}

This study obtained informed written consent from the patient authorizing publication of his clinical case and his photographs. His anonymity has been preserved. All investigations and interventions were in accordance with ethical standards.

\section{Consent for publication}


Written informed consent was obtained from the patient for publication of this case report and any accompanying images. A copy of the written consent is available for review by the Editor-in-Chief of this journal.

\section{Availability of data and materials}

The datasets used and/or analysed during the current study are available from the corresponding author on reasonable request.

\section{Competing interests}

The authors have no relevant financial or non-financial competing interests to disclose.

\section{Funding}

This project was supported by a Grant from Science and Technology of Jinan, China (No.202019003) .

\section{Authors' contributions}

Study concept and design: DWJ; Acquisition of data: LXY and XSS; Analysis and interpretation of data: LXY and XSS; Drafting of the manuscript: LXY; Critical revision of the manuscript for important intellectual content: XSS $₫$ Statistical analysis: LXY and XSS; Study supervision: DWJ; All authors read and approved the final manuscript.

\section{Acknowledgements}

Thanks to Dr. Edward C. Mignot, Shandong University, for linguistic advice.

\section{References}

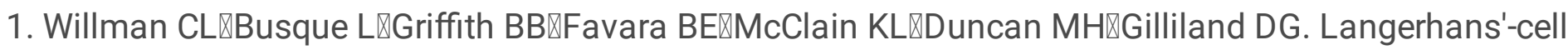
histiocytosis (histiocytosis X)-a clonal proliferative disease. N Engl J Med. (1994) 331:154-160. doi: 10.1056/NEJM199407213310303

2. Chang KL, Snyder DS. Langerhans cell histiocytosis. Cancer Treat Res. (2008) 142:383-398. doi: 10.1007/978-0-387-73744-7_17

3. Satter EK, High WA. Langerhans cell histiocytosis: a review of the current recommendations of the Histiocyte Society. Pediatr Dermatol. (2008) 25:291-5. doi: 10.1111/j.1525-1470.2008.00669.x

4. Arico M, Girschikofsky M, Genereau T, Klersy C, McClain K, Grois N, Emile JF, Lukina E, De JE, Danesino C. Langerhans cell histiocytosis in adults. Report from the International Registry of the Histiocyte Society. Eur J Cancer. (2003) 39: 2341-2348. doi: 10.1016/s0959-8049(03)00672-5

5. Egeler RM, Favara BE, Van M, Laman, JD, Claassen E. Differential In situ cytokine profiles of Langerhans-like cells and T cells in Langerhans cell histiocytosis: abundant expression of cytokines relevant to disease and treatment. (1999) 94: 4195-4201. doi:10.1159/000080864 
6. Broadbent V, Gadner H, Komp DM, Ladisch S. Histiocytosis syndromes in children: II. Approach to the clinical and laboratory evaluation of children with Langerhans cell histiocytosis. Med Pediatr Oncol. (1989) 17: 492-495. doi: 10.1002/mpo.2950170527

7. Thompson HH, Pitt HA, Lewin KJ, Longmire WJ. Sclerosing cholangitis and histiocytosis X. Gut. (1984) 25: 526-530. doi: 10.1136/gut.25.5.526

8. Ramos FJ, Perez-Arellano JL, Lopez-Borrasca A. Primary sclerosing cholangitis in histiocytosis X. Am J Med. (1987) 82: doi: 10.1016/0002-9343(87)90419-0

9. Satter EK, High WA. Langerhans cell histiocytosis: a review of the current recommendations of the Histiocyte Society. Pediatr Dermatol. (2008) 25: 291-295. doi:10.1111/j.1525-1470.2008.00669.x

10. Calming U, Henter JI. Elevated erythrocyte sedimentation rate and thrombocytosis as possible indicators of active disease in Langerhans' cell histiocytosis. Acta Paediatr. (1998) 87: 1085-1087. doi: $10.1080 / 080352598750031437$

11. Caputo R, Marzano AV, Passoni E, Fassati LR, Agnelli F. Sclerosing cholangitis and liver transplantation in Langerhans cell histiocytosis: a 14-year follow-up. Dermatology. (2004) 209: 335337. doi: $10.1159 / 000080864$

12. Braier J, Ciocca M, Latella A, Davila MG, Drajer M, Imventarza O. Cholestasis, sclerosing cholangitis, and liver transplantation in Langerhans cell Histiocytosis. Med Pediatr Oncol. 38: 178-182. doi: 10.1002/mpo.1306

13. Braier J, Ciocca M, Latella A, Davila MG, Drajer M, Imventarza O. Cholestasis, sclerosing cholangitis, and liver transplantation in Langerhans cell Histiocytosis. Med Pediatr Oncol. 38: 178-182. doi: 10.1002/mpo.1306

\section{Table}

Table 1 Timeline of clinical case history, time refers to the initiation of treatment with famotidine (week = 0)

\begin{tabular}{lc}
\hline Event & Week \\
\hline Lymphocytic thyroiditis & -192 \\
Fatigue and jaundice & -48 \\
Nails keratinize & -48 \\
Sclerosing cholangitis & 0 \\
Langerhans cell histiocytosis & $+1-2$ \\
Etoposide and prednisolone & $+3-27$ \\
VP-16, Prednisone acetate, cyclophosphamide, vinblastine & $+28-76$ \\
\hline
\end{tabular}

\section{Figures}



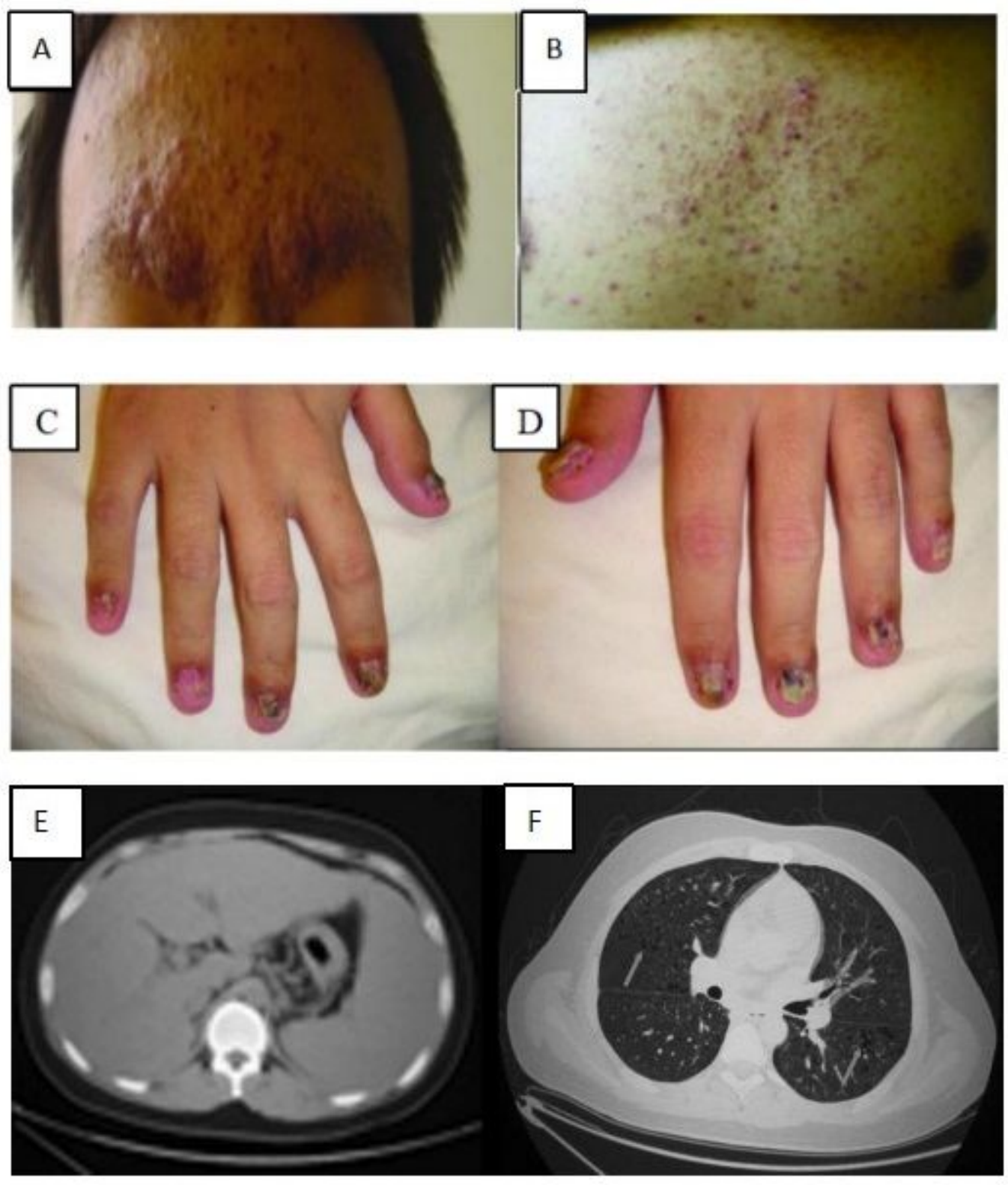

\section{Figure 1}

1A. The facial skin is present with extensive coalescing, scaling, or crusted papules. 1B Skin lesions in trunk. 1C Onycholysis, loss of nail plate, subungual hyperkeratosis of his fingernails in right hand; 1D Nails lesions in left hand. 1E. Computed tomography (CT) showing enlargement of lymphnodes, lowdensity node of liver. 1F. Computed tomography (CT) showing multiple cystic pulmonary lesions indicating pulmonary LCH ( Arrow target). 

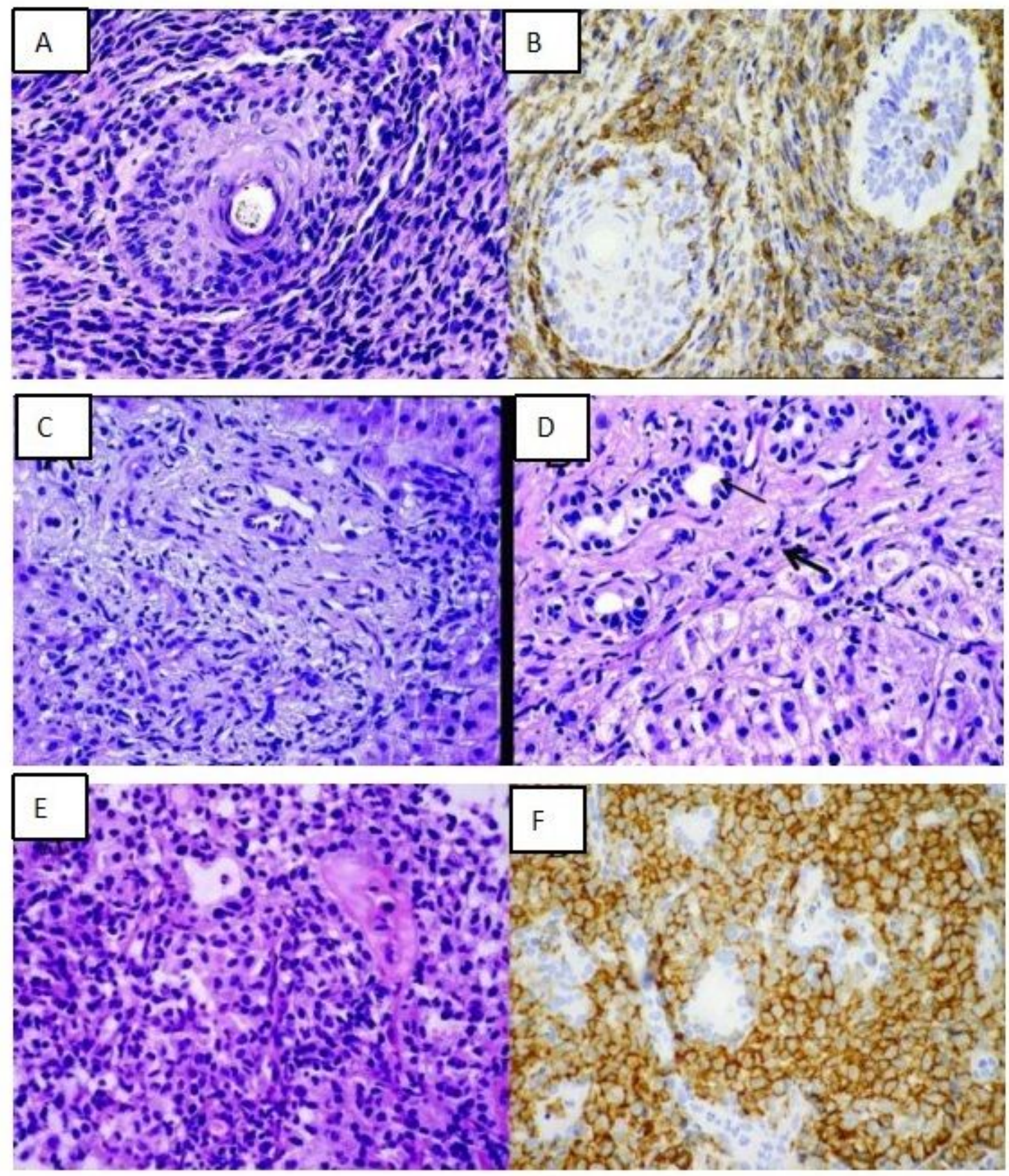

Figure 2

2A The hepatic biopsy showing irregular bile duct wall, loss of bile ductile and periductal fibrodis; $2 \mathrm{~B}$. Proliferation of ductile, enlarged bile duct(pyknic Arrow figure), infiltration of intestinal cells and mild periductal fibrosis in liver biopsy. 2C The skin granuloma consisting predominantly of macrophages, with eosinophils intermixed; 2D Immunohistochemical stain-the macrophages showing strong positivity to antibodies against CD1a antigen and S-100 protein. 2E. Around the thyroid gland lumina is infiltrated with macrophage cells. 2F. Positive CD1a and S-100 immunohistochemical staining. 